\title{
El uso de la red social facebook para fortalecer en los alumnos la obtención de información y expresión de las ideas
}

The use of social network facebook to strengthen students in obtaining information and expressing ideas

José Juan Miranda Torres

Escuela Preparatoria 1"Colegio Civil", Universidad Autónoma de Nuevo León.

mirtojj@hotmail.com

\section{Resumen}

La investigación se centra en las insuficiencias por parte de los estudiantes en el manejo de las TIC, con el objetivo de contribuir al desarrollo de competencias en la búsqueda de información mediante las TIC. La idea a defender: está dada porque la orientación de los estudiantes en el empleo de la red social Facebook, desde las actividades docentes permitirá aprovechar la novedad del medio para lograr que los estudiantes obtengan información adecuada y mejoren en la expresión de sus ideas. La investigación se desarrolla utilizando los métodos teóricos y empíricos de la investigación científica. El aporte prácticos está dado por la estrategia didáctica: "El uso de la red social Facebook para fortalecer en los alumnos el manejo de las TIC'S en la obtención de información y expresión de las ideas", la misma se enmarca en la unidad de aprendizaje: Tecnologías de la Información y la Comunicación I, perteneciente al campo disciplinar de Comunicación, Área Curricular Básica, modalidad presencial, primer semestre de la Preparatoria \# .1 de la Universidad Autónoma de Nuevo León, México. El resultado es el diseño argumentado y comunicable de la estrategia didáctica propuesta.

Palabras claves: facebook, redes sociales, búsqueda de información. 


\section{Abstract}

The research focuses on the inadequacies on the part of students in the management of ICT, with the aim of contributing to the development of skills in searching for information using ICT. The idea to defend: is given for the guidance of students in the use of social network Facebook, from educational activities will take the novelty of the medium to ensure that students get adequate information and enhance the expression of their ideas. The research is developed using theoretical and empirical methods of scientific inquiry. The practical contribution is given by the teaching strategy: "The social network use Facebook to strengthen the students' ICT skills'S in obtaining information and expression of ideas," it is part of the unit learning Information Technology and Communication I, belonging to the discipline of Communication, Basic Academic Area, modality, first semester of School \# 1 at the Autonomous University of Nuevo Leon, Mexico. The result is the design and communicable argued the proposed teaching strategy.

Key words: facebook, social networking, searching for information.

Fecha recepción: Agosto 2012

Fecha aceptación: Diciembre 2012

\section{Introducción}

Durante décadas la estrategia educativa en México estuvo centrada en expandir la educación primaria y secundaria, ese fue el gran desafío en el siglo XX, en el que nuestro país logro abatir en su totalidad el analfabetismo y la carencia educativa. Tuvimos por más de 40 años un país de niños y los esfuerzos se centraron en ese sector.

El gran desafío del siglo XXI es enfrentar un país con una demografía distinta, en la que la mayoría de la población es de jóvenes y ya no de niños y donde el problema de la educación básica está resuelto. El bachillerato en México presenta problemas históricos, problemas que tienen que ver con la calidad del servicio educativo, con la cobertura, y con la equidad. A menor ingreso ha existido una menor capacidad para enviar a los hijos a la escuela, lo que ha reducido la posibilidad de que las clases populares aspiren a educación para sus hijos. 
De la misma forma existe una deserción significativa en la educación media. El $45 \%$ de quienes abandonan la escuela, esto es casi la mitad de quienes ingresan a 10. De primaria lo hacen entre el primero y tercero de preparatoria. Esto incide en una pobre eficiencia terminal, apenas termina el $55 \%$ de los que se inscribieron en 10 de primaria hasta el bachillerato.

Cuando comparamos el desempeño de México en relación con los avances educativos en otros países encontramos un preocupante rezago educativo en México, no solo en el promedio de años que un estudiante permanece en la escuela en Europa o Norteamérica, sino sobre todo en la calidad educativa.

Las causas de abandono están efectivamente en múltiples razones de índole socioeconómicas, de necesidades de sustento familiares, de inserción temprana al mercado laboral, pero también hay razones referentes al interés y a la pertinencia. Los jóvenes expresan en un porcentaje considerable que no se les hace interesante y atractivo para su vida cotidiana estudiar. Si bien esta medido que a mayor estudio se tiene mayor ingreso, los estudiantes parecen no saberlo o creerlo o incluso ignorarlo, esto refleja con claridad que el tipo de educación que se les ofrece no les sirve para enfrentar el mundo real, el campo de trabajo.

Esa falta de interés o de atracción y compromiso tiene que ver con los contenidos educativos y con la organización del Sistema de Educación Media Superior. Existen 25 subsistemas de distintas formaciones, estructuras, duraciones y especialidades cuyo tránsito, acreditación o revalidación entre unas y otras es extremadamente complicado.

Se han detectado casos en los que ha sido más fácil revalidar un estudiante extranjero que un estudiante que sale de un CBTIS y pretende inscribirse a un CANALEP.

Ante esta problemática los retos y desafíos del mundo contemporáneas crecen día a día , hay cambios continuos en los esquemas de convivencia social y participación política, medios de comunicación, la tecnología que hoy inunda nuestra vida cotidiana y en consecuencia el mercado laboral y en estos cambios las tecnologías están jugando un papel protagónico.

Exigencias de más elevada calidad, competencia, conocimiento y experiencia son demandadas a muy temprana edad a la par capacidades innovadoras como el desempeño en ambientes plurales, virtuales, actitudes críticas, universos informativos al alcance de la mano y adaptabilidad continua.

Los jóvenes del siglo XXI toman decisiones trascendentes, en escenarios cambiantes justamente en la etapa que cursan el bachillerato. Por lo que resulta significativo el trabajo didáctico que se realice 
para reforzar todo lo relacionado con el manejo de las TIC'S en la obtención de información y expresión de las ideas.

Este es el origen de la Reforma, la necesidad de un cambio, de corregir los errores, de ponernos al día con el mundo, pero sobre todo de mejorar, de elevar los niveles y la calidad, de contar con un sistema que responda más a las distintas realidades de los jóvenes de México y a las necesidades del mundo laboral.

Uno de los ejes en que se desarrolla esta Reforma Integral de la Educación Media Superior (RIEMS), de México, es la instrumentación de mecanismos de gestión que favorezcan el desarrollo de la RIEMS. Estos mecanismos definen estándares y procesos comunes que contribuyen al desarrollo de las competencias genéricas y disciplinares básicas. Según lo anterior, la instrumentación de estos mecanismos presupone tener en cuenta las características propias de los estudiantes de bachilleratos y en este sentido se conoce que los jóvenes de 15 a 19, por lo general y al menos en el ámbito urbano, se encuentran en una etapa del desarrollo caracterizada, desde el punto de vista psicosocial, por una creciente autonomía con respecto al núcleo familiar, un fortalecimiento de redes sociales propias, y un incremento de los intereses personales extrafamiliares y extraescolares, todas ellas circunstancias fértiles para el surgimiento de culturas juveniles, claramente diferenciadas del mundo adulto. (SEMS, 2008).

Esta etapa se caracteriza, además, por la toma de decisiones en relación al proyecto de vida (migración, trabajo, matrimonio, otros), lo que desplaza el interés y la atención de los jóvenes hacia un abanico de áreas que compite fuertemente con la motivación académica. Es por eso que la posibilidad de logro escolar se incrementará si las trayectorias de aprendizaje, de los diferentes contenidos objeto de estudio, atienden a la generación de espacios de orientación educativa, de la forma más conveniente, procurando que el desarrollo integral y equilibrado que caracteriza la educación básica se extienda hasta el nivel medio superior. (SEMS, 2008).

Muchos de los espacios que pueden ser generados se basan en la utilización de herramientas tecnológicas, entre las que se pueden mencionar a los e-portfolios o portafolios digitales, las redes sociales y los documentos y aplicaciones en la red o Cloud Computing.

La proliferación de tecnologías participativas y colaborativas, como son las redes sociales, es un hecho y los jóvenes juegan un papel protagónico en esta proliferación. Según un estudio realizado por la 
AMIPCI seis de cada diez internautas mexicanos, es usuario de alguna red social; la mayoría prefiere: Facebook, Youtube, Twitter.

En este contexto, no se puede obviar el impacto que las redes sociales tiene entre los jóvenes, sin embargo aún es insuficiente el trabajo que hacen los profesores para aprovechar las ventajas de las redes sociales para fortalecer en los alumnos el manejo de las TIC'S en la obtención de información y expresión de las ideas, estimular su actividad de estudio y propiciar el desarrollo de competencias. Teniendo en cuenta los aspectos anteriores se precisa el diseño metodológico de la investigación:

\section{Desarrollo}

La propuesta está ajustada al contexto donde se encuentra enclavada la Preparatoria No.1 de la UANL, que es el municipio de Apodaca, el cual se caracteriza por un incremento considerable en su demografía e industria, ambos aspectos requieren de jóvenes bien preparados y competentes en el uso de las Tecnologías de la Información y la Comunicación, para poder desempeñarse con éxito en la sociedad del conocimiento. Se basa en varios documentos e instrumentos de análisis de contexto y de fundamentación teórica para determinar áreas de oportunidad y/o detección de necesidades académicas del grupo. El grupo en donde será aplicada la estrategia didáctica consta de 38 alumnos, 22 mujeres y 16 hombres. Para conocer algunos aspectos acerca de la participación de los padres de familia en la educación de sus hijos, se aplicó una encuesta en la que se evidencia que el $76 \%$ de los padres de estos alumnos tienen un nivel educativo de preparatoria o licenciatura; y el $37 \%$ está al pendiente de las tareas y actividades que realizan. En cuanto a los antecedentes de los estudiantes, se utilizó un instrumento institucional de la Universidad Autónoma de Nuevo León (UANL) denominado Índice de Predicción del Rendimiento (IPRE) en donde los resultados indicaron que el grupo muestra deficiencias en las áreas de Orden y Voluntad para la realización de tareas, con un $7.1 \%$ y $7.2 \%$ respectivamente.

Esta problemática concuerda con la establecida en el Acuerdo 442 (2008) en donde se señala que:

Los jóvenes de 15 a 19, por lo general y al menos en el ámbito urbano, se encuentran en una etapa del desarrollo caracterizada, desde el punto de vista psicosocial, por una creciente autonomía con respecto al núcleo familiar, un fortalecimiento de redes sociales propias, y un incremento de los intereses personales extrafamiliares y extraescolares, todas ellas circunstancias fértiles para el surgimiento de culturas juveniles, claramente diferenciadas del mundo adulto. (p. 82) 
Con la intención de conocer el uso de la red social Facebook que realizan los alumnos en su vida diaria o en actividades escolares, se aplicó una encuesta. Los resultados son como sigue: ¿Tienes una cuenta de Facebook? El 100\% dice que SI. ¿Utilizas la red social Facebook para comunicarte con tus amigos frecuentemente? El 97\% de los alumnos menciona que SI, mientras que el 3\% restante dice que NO y en el apartado de comentarios, puntualizan que lo realizan esporádicamente. ¿Los docentes de las distintas unidades de aprendizaje utilizan la red social Facebook como herramienta para que desarrolles actividades de aprendizaje en sus clases? El 100\% menciona que NO ¿Te gustaría utilizar la red social Facebook para discutir y hacer aportaciones sobre temas específicos en las distintas materias que cursas este semestre? El $100 \%$ de los estudiantes menciona que SI.

La estrategia didáctica: "El uso de la red social Facebook para fortalecer en los alumnos el manejo de las TIC'S en la obtención de información y expresión de las ideas"se enmarca en la unidad de aprendizaje: Tecnologías de la Información y la Comunicación I, perteneciente al campo disciplinar de Comunicación, Área Curricular Básica, modalidad presencial, primer semestre. "Las competencias disciplinares básicas de comunicación están referidas a la capacidad de los estudiantes de comunicarse efectivamente en español...mediante el uso de distintos medios e instrumentos". Además, "usarán las tecnologías de la información y la comunicación de manera crítica para diversos propósitos comunicativos" (Acuerdo 444, 2008, 7). (Anexo 6)

Para el logro de la estrategia, se pretende desarrollar la competencia disciplinar:

12. Utiliza las tecnologías de la información y la comunicación para investigar, resolver problemas, producir materiales y transmitir información.

Se reforzarán en los alumnos a través de la estrategia didáctica, las competencias genéricas:

4. Escucha, interpreta y emite mensajes pertinentes en distintos contextos mediante la utilización de medios, códigos y herramientas apropiados.

Maneja las tecnologías de la información y la comunicación para obtener información y expresar ideas.

5. Desarrolla innovaciones y propone soluciones a problemas a partir de métodos establecidos.

> Utiliza las tecnologías de la información y comunicación para procesar e interpretar información.

En el México de hoy es indispensable que los jóvenes que cursan el bachillerato egresen con una serie de competencias que les permitan desplegar su potencial, tanto para su desarrollo personal como 
para contribuir al de la sociedad. Las competencias comunes a todos los egresados de la EMS son las competencias genéricas, el Acuerdo 444 menciona:

Son competencias clave, por su importancia y aplicaciones diversas a lo largo de la vida; transversales, por ser relevantes a todas las disciplinas y espacios curriculares de la EMS, y transferibles, por reforzar la capacidad de los estudiantes de adquirir otras competencias. (p.2)

En el desarrollo del Perfil de Egreso del alumno de bachiller a través de las competencias genéricas y disciplinares, la labor del docente es fundamental. En el Acuerdo 447 en el 2008, al respecto menciona que:

Es indispensable que los maestros trasciendan los propósitos exclusivamente disciplinares y apoyen de manera integral la formación de los jóvenes; que vaya más allá de las prácticas tradicionales de enseñanza en el salón de clases, para adoptar un enfoque centrado en el aprendizaje en diversos ambientes, sobre todo ante la Reforma Integral de la Educación Media Superior emprendida para el establecimiento del Sistema Nacional de Bachillerato (SNB) en un marco de diversidad. (p.1)

La Secretaría de Educación Pública define el Perfil del Docente del Sistema Nacional de Bachillerato, el cual está constituido por un conjunto de competencias para quienes imparten educación media superior en la modalidad escolarizada, las que integran conocimientos, habilidades y actitudes que el docente pone en juego para generar ambientes de aprendizaje en los que los estudiantes desplieguen las competencias genéricas. En el Acuerdo 444 (2008) "Las competencias docentes son las que formulan las cualidades individuales, de carácter ético, académico, profesional y social que debe reunir el docente de la EMS, y consecuentemente definen su perfil" (p.1).

Para desarrollar la estrategia didáctica "El uso de la red social Facebook para fortalecer en los alumnos el manejo de las TIC'S en la obtención de información y expresión de las ideas" el docente requiere las siguientes competencias:

3. Planifica los procesos de enseñanza y de aprendizaje atendiendo al enfoque por competencias, y los ubica en contextos disciplinares, curriculares y sociales amplios.

$>$ Identifica los conocimientos previos y necesidades de formación de los estudiantes y desarrolla estrategias para avanzar a partir de ellas.

$>$ Contextualiza los contenidos de un plan de estudios en la vida cotidiana de los estudiantes y la realidad social de la comunidad a la que pertenecen. 
4. Lleva a la práctica procesos de enseñanza y de aprendizaje de manera efectiva, creativa e innovadora a su contexto institucional.

> Utiliza la tecnología de la información y la comunicación con una aplicación didáctica y estratégica en distintos ambientes de aprendizaje.

5. Evalúa los procesos de enseñanza y de aprendizaje con un enfoque formativo.

> Establece criterios y métodos de evaluación del aprendizaje con base en el enfoque de competencias, y los comunica de manera clara a los estudiantes.

$>$ Se mantiene actualizado en el uso de la tecnología de la información y la comunicación.

Para lo que se debe tener en cuenta que la evaluación por competencias presupone:

- PRINCIPIO 1: La evaluación de las competencias es un proceso metacognitivo

- PRINCIPIO 2: La evaluación se basa en criterios pertinentes al desempeño en el contexto

- PRINCIPIO 3: La evaluación busca articular lo cualitativo y lo cuantitativo

- PRINCIPIO 4: La evaluación se centra en los aspectos esenciales del aprendizaje

- PRINCIPIO 5: La retroalimentación motiva al mejoramiento continuo

- PRINCIPIO 6: La evaluación es intersubjetiva, dialógica y tiene control de calidad

Y que los pasos esenciales de la evaluación son:

\section{Pasos esenciales en la evaluación}

PASO 1: ¿Qué evaluar?

PASO 2: ¿Para qué evaluar?

PASO 3: ¿Con qué criterios?

PASO 4: ¿Con qué pruebas?
Saberes de la

competencia

Para formar y valorar la idoneidad

Logros esperados en la competencia

Evidencias concretas de aprendizaje 


\section{E1 proceso de evaluación paso a paso}

\author{
PASO 5: ¿Cómo \\ determinar el nivel \\ de aprendizaje? \\ PASO 6: ¿En qué \\ momentos evaluar? \\ PASO 7: ¿Con \\ qué estrategias? \\ PASO 8: ¿Cómo \\ informar?
}

Matrices de evaluación

Diagnóstico, Formativa, Promoción y Acreditación

Pruebas, portafolio, observación, simulaciones, etc.

Logros, aspectos a mejorar y nivel de aprendizaje

\section{- el PRINCIPIO de “ALINEACIÓN CONSTRUCTIVA COMO SUSTENTO de la ESTRATEGIA PROPUESTA:}

La estrategia que propongo se basa en el concepto de alineación constructivista, el cual recomienda que para un buen diseño de estrategias de aprendizaje debemos alinear lo que se pretende evaluar con los objetivos o metas de aprendizaje y enfocar las actividades o productos de los estudiantes hacia el dominio de las competencias expresadas en los atributos (Biggs, 2010).Los principios de aprendizaje que sirven de base en la propuesta didáctica en el enfoque en competencias, se fundamentan en una visión constructivista, según el Acuerdo 442 (2008):

(...) reconoce al aprendizaje como un proceso que se construye en forma individual, en donde los nuevos conocimientos toman sentido estructurándose con los previos y en su interacción social. Por ello, un enfoque de competencias conlleva un planteamiento pertinente de los procesos de enseñanza y aprendizaje, actividad que compete al docente, quien promoverá la creación de ambientes de aprendizaje y situaciones educativas apropiadas al enfoque en competencias, favoreciendo las actividades de investigación, el trabajo colaborativo, la resolución de problemas, la elaboración de proyectos educativos interdisciplinares, entre otros. De la misma manera, la evaluación de las competencias de los estudiantes requiere el uso de métodos diversos, por lo que los docentes deberán contar con las herramientas para evaluarlas. (p.46) 
Al preparar a nuestros alumnos con las competencias mínimas requeridas por el perfil de egreso de la Educación Media Superior, específicamente en los atributos en los que se especifica la competencia del alumno para el manejo de las Tecnologías de la Información y la Comunicación (4ạ y 5ạ competencia genérica), estaremos aportando mejoras a nuestro programa de la materia disciplinar dentro del Marco Curricular Común de la Reforma Integral en México.

El principio de "alineación constructiva" se desarrolló con la decisión de usar un "portfolio" para evaluar la medida por la cual los alumnos sintieron que habían satisfecho los objetivos de la unidad.

Esto los forzó a reflexionar sobre lo que deseaban de la misma, y sobre cómo podían obtenerlo, lo cual a su vez presionó sobre el docente para que les proporcionara adecuadas actividades de enseñanza-aprendizaje para ayudarlos a llevarlo a cabo.

De esta forma, todos los componentes en el sistema devinieron alineado a los objetivos.

La pregunta es: ¿Pueden los principios del alineamiento constructivo ser generalizados en relación con lo efectuado en esta experiencia?

La cuestión clave, es si el profesor puede operacionalizar convenientemente altos niveles de comprensión, de forma que denoten desempeños que pueden ser felicitados por adecuadas actividades de enseñanza-aprendizaje y que, además, puedan ser evaluados auténticamente.

Es entonces, una cuestión de aplicar los principios de la alineación y de la evaluación referida a criterios que ya están muy bien establecidos en la literatura del diseño de la instrucción.

A la crítica de que la evaluación referida a criterios es cerrada y enemiga del espíritu del constructivismo, se puede señalar al portfolio, en donde todo el apoyo es proporcionado a los estudiantes por ser divergente y sorpresivo.

El modelo de enseñanza que surge es simple.

- Los profesores deben ser claros acerca de lo que desean que sus alumnos aprendan y de qué manera manifestarán dicho aprendizaje en términos de desempeños que manifiesten su comprensión. Así, por ejemplo, memorizar y parafrasear no son desempeños de comprensión. Sí lo es efectuar una aplicación en un contexto nuevo.

- Los objetivos de desempeños que así emerjan, necesitan ser ordenados en una forma jerárquica desdelo más aceptable a lo apenas satisfactorio. En tal jerarquía, operará el sistema de calificación. 
- Los alumnos necesariamente deberán ser colocados en situaciones que se juzguen como más probables para felicitar los aprendizajes requeridos. De tal manera, se les requerirá que proporcionen evidencias, sean en tareas propuesta por el docente o por ellos, de modo que lo aprendido pueda ser apareado con los objetivos acordados. Su calificación será la más alta si tal comparación es efectuada de modo convincente.

De los buenos profesores, se espera que sean claros acerca de lo que desean que los estudiantes aprendan, y qué deberían hacer éstos a fin de demostrar que lo han logrado en un nivel adecuado.

Además, deberían conocer y poner en funcionamiento formas de lograr que sus alumnos aprendan de modo efectivo y a un nivel deseable de conocimientos. Igualmente, a ser más centrados en los estudiantes y en sus actividades de enseñanza-aprendizaje y más auténticos en sus evaluaciones.

El presente modelo proporciona un marco para operacionalizar sistemáticamente estos deseos. Existe, sin embargo, un lado institucional para esto, por no decir una obligación.

El enmarque cuantitativo del control institucional, tal como es efectuado en el modelo administrativo que recientemente se ha impuesto en universidades de muchos países, frecuentemente requiere de evaluaciones relacionadas con prácticas que tornan al tipo de enfoque cualitativo identificado como de referencias al criterio, difícil de aplicar [BIGGS, 1996]

La racionalidad económica, significa grandes clases, lo cual, en el marco del pensamiento convencional significa más sesiones de tipo conferencia y más exámenes finales especialmente del tipo opción múltiple, más bien que trabajos de grupo, asignaciones u otros formatos que consumen tiempo para calificar. Esto no

debe ser así necesariamente, pero es fácil de pensar por qué lo es. El pensamiento administrativo cada vez más requiere el informe de los desempeños de los estudiantes, en términos de porcentajes, más bien quede calificaciones expresadas en letras u otras categorías cualitativas.

En Australia, muchas instituciones aún requieren calificar bajo la curva. Tales enfoques desalientan marcadamente enfoques cualitativos a la evaluación de los alumnos.

Existe una real tensión entre los requerimientos administrativos y los académicos, las necesidades académicas devienen más proactivas, insistiendo positivamente en que las consideraciones educativas deben prevalecer sobre las conveniencias administrativas.

Sin embargo, estas consideraciones conducen a diferentes conjuntos de cuestiones, las cuales podría ser inapropiado continuarlas aquí. 
Mi posición es que, una versión operante del constructivismo puede ser integrada con el diseño instruccional en tres puntos cruciales:

1. Los objetivos del currículo de las unidades, deberán ser formuladas en términos de contenidos de específicos niveles de comprensión que impliquen desempeños apropiados.

2. Los métodos de enseñanza requieren que los alumnos sean colocados en contextos con más probabilidad de felicitar estos desempeños o actuaciones.

3. Las actividades de evaluación serán orientadas a considerar tales desempeños o actuaciones. A opinión del autor esta concepción proporciona un poderoso contexto de enseñanza-aprendizaje.

\section{DISEÑO ARGUMENTADO Y COMUNICABLE DE LA ESTRATEGIA DIDÁCTICA PROPUESTA.}

\section{- FUNDAMENTACIÓN}

Los principios de aprendizaje que sirven de base en la propuesta didáctica: "El uso de la red social Facebook para fortalecer en los alumnos el manejo de las TIC'S en la obtención de información y expresión de las ideas"en el enfoque en competencias, se fundamentan en una visión constructivista, según el Acuerdo 442 (2008):

(...) reconoce al aprendizaje como un proceso que se construye en forma individual, en donde los nuevos conocimientos toman sentido estructurándose con los previos y en su interacción social. Por ello, un enfoque de competencias conlleva un planteamiento pertinente de los procesos de enseñanza y aprendizaje, actividad que compete al docente, quien promoverá la creación de ambientes de aprendizaje y situaciones educativas apropiadas al enfoque en competencias, favoreciendo las actividades de investigación, el trabajo colaborativo, la resolución de problemas, la elaboración de proyectos educativos interdisciplinares, entre otros. De la misma manera, la evaluación de las competencias de los estudiantes requiere el uso de métodos diversos, por lo que los docentes deberán contar con las herramientas para evaluarlas. (p.46)

La fundamentación teórica de la estrategia didáctica que se propone está dada por los postulados de Biggs (2010), el que se ubica en un paradigma constructivista, que asume que se aprende cuando un alumno afronta distintas actividades de aprendizaje, pero en este contacto el alumno puede realizar actividades profundas, que impliquen crear nuevas estructuras con los contenidos, o por el contrario, realizar actividades superficiales que únicamente supongan un procesamiento superficial de la información, con una acumulación sumarial de datos sin estructurar. 
El Impacto de la Reforma en la atención de la problemática detectada en mi contexto de trabajo, radica en el despliegue de la propuesta didáctica, que se centra principalmente en el desarrollo de una serie de estrategias de aprendizaje que culminan con la realización de un debate grupal a través de la red social FaceBook, la que tiene gran aceptación y uso entre los jóvenes de estas edades. La intención es aprovechar el interés que los alumnos de la Preparatoria No. 1 muestran por esta herramienta electrónica de comunicación social, para que a través de este medio, expresen sus ideas y conocimientos adquiridos referentes a los elementos que conforman un sistema computacional.Los aprendizajes en la Educación Media Superior deben ser significativos para los estudiantes. Al respecto, en el Acuerdo 442 (2008) se menciona que, cuando los jóvenes reconocen en su vida cotidiana y en sus aspiraciones las ventajas de lo que aprenden en la escuela, redoblan el esfuerzo y consolidan los conocimientos y las habilidades adquiridas, esto conducirá a elevar la cobertura y permanencia en la EMS, en tanto que los estudiantes advertirán las ventajas que representa continuar sus estudios.En el despliegue de la estrategia didáctica "El uso de la red social Facebook para fortalecer en los alumnos el manejo de las TIC'S en la obtención de información y expresión de las ideas"el docente, dará prioridad al trabajo cooperativo (Acuerdo 442, 2008) ya que con ello:

...no solamente se dinamizan los conocimientos declarativos y procedimentales de los alumnos, también se movilizan habilidades sociales y de convivencia, así como actitudes y valores tales como respeto y tolerancia hacia las ideas de los demás, responsabilidad individual y compartida, habilidades de comunicación, intercambio de información, negociaciones y acuerdos sociales, desarrollo de buenas relaciones, habilidad para solucionar conflictos, entre otros. (p.13)

El enfoque pedagógico utilizado en el diseño de las actividades de aprendizaje se fundamenta en las 5 dimensiones de aprendizaje de Marzano. De acuerdo a lo anterior Chan (2002) propone que las actividades de aprendizaje deben cubrir un proceso de aprendizaje dinámico a través del cual los estudiantes serán capaces de construir sus aprendizajes.

En relación a la secuencia didáctica de la propuesta, se está considerando el modelo de Alfonzo (2003, citado por Ronald Feo) en el que se considera tres momentos: Momento de inicio, para contar con una visión preliminar del tema; Momento de desarrollo, en el que se utiliza la estrategia de enseñanza y aprendizaje, y el Momento de cierre, que permite resumir el tema, transferir el aprendizaje y hacer el cierre. 
En cuanto a la evaluación utilizada en la estrategia didáctica: "El uso de la red social Facebook para fortalecer en los alumnos el manejo de las TIC'S en la obtención de información y expresión de las ideas" está basada en el Acuerdo 8 (2009) en el que: "se relaciona a la generación de evidencias sobre los aprendizajes asociados al desarrollo progresivo de las competencias que establece el marco Curricular Común" (p.2).

Asimismo, según el Acuerdo 8 (2009): "es necesario tener en cuenta la diversidad de formas y ritmos de aprendizaje de los alumnos, para considerar que las estrategias de evaluación atiendan los diferentes estilos de aprendizaje" (p.2)

Según el momento de la evaluación (Acuerdo 8, 2009) en la estrategia didáctica, se realiza:

- Evaluación diagnóstica, se realiza para estimar los conocimientos previos de los estudiantes que ayuden a orientar el proceso educativo.

- Evaluación formativa, permite precisar los avances logrados por cada alumno y, de manera especial, advertir las dificultades que encuentra durante el aprendizaje; tiene por objeto mejorar, corregir o reajustar el avance del alumno. Implica una reflexión y un diálogo con los alumnos acerca de los resultados obtenidos y los procesos de aprendizaje y de enseñanza que los llevaron a ellos; permite estimar la eficacia de las experiencias de aprendizaje para mejorarlas y en el alumno favorece el desarrollo de su autonomía. (p.3)

En la estrategia de evaluación global utilizada en la propuesta, el diálogo forma parte esencial del debate. Para Avolio de Cols e lacoluttti (2006 citado por Diana Dolores Janitzio de León Cerda) "el diálogo es una técnica que permite evaluar la capacidad de reflexión sobre la práctica en el desarrollo del proceso y de los criterios puestos en juego" (p.31)

La realización del debate, permite al docente completar su evaluación con otras evidencias, también posibilita la participación directa del estudiante en el proceso, así mismo, le da la posibilidad de comprender, aprender e integrar lo que está detrás de su desempeño, se puede decir que es la competencia puesta en acción. Se utilizará una rúbrica contenida en el Anexo 4 y se evalúa al final a cada alumno su participación en el foro de manera integral.

\section{- DISEÑO DE LA ESTRATEGIA DIDÁCTICA.}

La estrategia didáctica: "El uso de la red social Facebook para fortalecer en los alumnos el manejo de las TIC'S en la obtención de información y expresión de las ideas" se ajusta al contexto donde se ubica la Preparatoria No.1 de la UANL: el municipio de Apodaca; el cual se caracteriza por un incremento 
considerable en su demografía e industria, esto requiere de jóvenes bien preparados y competentes en el uso de las Tecnologías de la Información y la Comunicación, para poder desempeñarse con éxito en su comunidad y en una sociedad del conocimiento.

Esta propuesta se enmarca en la Unidad de Aprendizaje Tecnologías de la Información y la Comunicación I, perteneciente al campo disciplinar de Comunicación, Área Curricular Básica, modalidad presencial, primer semestre. "Las competencias disciplinares básicas de comunicación están referidas a la capacidad de los estudiantes de comunicarse efectivamente en español...mediante el uso de distintos medios e instrumentos". Además, "usarán las tecnologías de la información y la comunicación de manera crítica para diversos propósitos comunicativos" (Acuerdo 444, 2008, 7)

La estrategia didáctica propuesta se va a aplicar en el grupo 3 de 1er. Semestre del Bachillerato General en el turno matutino, el cual está formado por 38 alumnos. La duración total de la misma, será aproximadamente de 12 sesiones de 50 minutos, 6 de ellas presenciales y el resto no presenciales.

A continuación se describe detalladamente la estrategia didáctica planteada: "El uso de la red social Facebook para fortalecer en los alumnos el manejo de las TIC'S en la obtención de información y expresión de las ideas" a través de una secuencia de actividades que se encuentran alineadas con el propósito, las competencias a desarrollar, los productos de aprendizaje y la evaluación planteada.

Propósito: "El alumno desarrolla las habilidades necesarias para el uso adecuado y eficiente de las tecnologías de la información y comunicación, como elemento esencial para informarse y comunicarse con una actitud ética".

Para el logro de la estrategia: "El uso de la red social Facebook para fortalecer en los alumnos el manejo de las TIC'S en la obtención de información y expresión de las ideas", se pretende desarrollar la Competencia Disciplinar:

12. Utiliza las tecnologías de la información y la comunicación para investigar, resolver problemas, producir materiales y transmitir información.

Se reforzarán en los alumnos a través de la estrategia didáctica, las Competencias Genéricas:

4. Escucha, interpreta y emite mensajes pertinentes en distintos contextos mediante la utilización de medios, códigos y herramientas apropiados.

> Maneja las tecnologías de la información y la comunicación para obtener información y expresar ideas. 
5. Desarrolla innovaciones y propone soluciones a problemas a partir de métodos establecidos.

$>$ Utiliza las tecnologías de la información y comunicación para procesar e interpretar información.

El tema de la estrategia didáctica es el Hardware. La competencia a desarrollar es: Identifica el hardware de un sistema computacional. Los contenidos que serán abordados en el desarrollo de la misma son:

$\checkmark$ Contenidos Conceptuales (saberes):

Los componentes de un sistema computacional: Dispositivos de Entrada (teclado, mouse, micrófono, scanner, webcam). Dispositivos de Salida (impresora, monitor y bocinas). Dispositivos de almacenamiento (memoria principal y memoria secundaria) y Dispositivos de Procesamiento (CPU)

$\checkmark$ Contenidos Procedimentales (habilidades):

Estructura de manera clara las ideas, las argumenta de manera coherente y sintética; considerando las reglas gramaticales y ortográficas. Realiza procedimientos mentales como: el análisis, la deducción y la síntesis de la información contemplada. Participa en el Foro a través de la red social Facebook en un debate en el cual opinan sobre el tema, lo defienden y argumentan su postura, enriqueciendo las opiniones de los compañeros

$\checkmark$ Contenidos Actitudinales (actitudes y valores):

Se interesa por aprender de sus compañeros. Participa de manera responsable en el foro, aportando puntos de vista. Contribuye con información para el trabajo en equipo. Realiza el trabajo en el tiempo asignado y en la forma indicada.

\section{- SECUENCIA DIDÁCTICA}

\begin{tabular}{l}
\hline SECUENCIA DIDÁCTICA \\
\hline$\underline{\text { INICIO }}$ \\
DIMENSIÓN: Problematización \\
Tiempo aproximado:1 sesión presencial de $\mathbf{5 0}$ minutos \\
Actividad del docente: El docente define al grupo la meta de aprendizaje "Identifica el \\
hardware de un sistema computacional". \\
Actividad del alumno: Los participantes expresan sus expectativas sobre el tema y resuelven \\
el examen diagnóstico (Anexo 1)
\end{tabular}


Producto: Resultado de la aplicación del Diagnóstico. Anexo 1

\section{DESARROLLO}

DIMENSIÓN: Adquisición del conocimiento y Procesamiento y organización de la información Tiempo aproximado:3 sesiones presenciales de 50 minutos

Espacio: Información. El alumno adquiere los insumos necesarios sobre el hardware (conceptos, premisas, procedimientos de uso, etc.) que le serán útiles permitiéndole en temas siguientes la comparación entre distintos tipos de dispositivos.

Espacio: Interacción. El alumno interactúa con la computadora e internet y en el Foro de discusión en la red social Facebook.

Actividad del docente:

- Indica a los alumnos que realicen la Actividad 1.2 pág. 17 de la Guía de aprendizaje Tecnología de la Información y la Comunicación 1. El docente realiza retroalimentación a los alumnos.

- Pide que investiguen en internet, visiten la página web que está disponible en las computadoras del Laboratorio de Cómputo de nuestra preparatoria, los elementos que conforman un sistema computacional y describa los elementos fundamentales así como las características de cada uno de ellos.

Actividad del alumno:

- Investiga en internet, visita la página web que está disponible en las computadoras del Laboratorio de Cómputo de nuestra preparatoria, los elementos que conforman un sistema computacional y describe los elementos fundamentales así como las características de cada uno de ellos.

- Realiza la lectura del tema: "Elementos fundamentales", de la unidad 1 de su libro de texto pág. 16-18.

- Elabora una lista en la que incluya todos los elementos, su descripción y su imagen.

Producto: Lista de elementos fundamentales de un sistema computacional: características e imagen, realizado en la libreta.

\section{CIERRE}

DIMENSIONES: Aplicación de la información y Metacognición

Tiempo aproximado: 8 sesiones 2 de ellas presenciales 
Espacio: Producción. Un mapa conceptual elaborado en Powerpoint.

Espacio: Exhibición. En la red social Facebook en un debate de los alumnos los cuales opinan sobre el tema, lo defienden y argumentan su postura, enriqueciendo las opiniones de los compañeros.

\section{Actividad del docente:}

- El docente realiza una dinámica para formar 7 equipos con 5 o 6 integrantes cada uno. Asigna a cada alumno del grupo un número del 1 al 7 de manera verbal. Pide que se coloquen en círculo todos alumnos cuyos números sean iguales, es decir que un círculo de alumnos serán "los unos", otro serán "los dos", y así sucesivamente hasta "los siete". De esta manera tendremos que los equipos del 1 al 3 estarán conformados por 6 integrantes y del 4 al 7 de 5 integrantes.

- El docente indica a los alumnos que realicen un mapa conceptual en equipo, considerando todos los elementos de un sistema computacional y realizarlo en una presentación en Powerpoint.

- El docente presenta las preguntas detonantes en la red social Facebook (Anexo 2), las que iniciarán un debate entre los alumnos, los cuales expresarán sus opiniones sobre el tema, lo defenderán y argumentarán su postura y realizar aportaciones para enriquecer las opiniones de sus compañeros. Además aclara a los alumnos que la participación se realizará con respeto hacia las opiniones de los demás y que por esta ocasión, se permitirá el uso del código acostumbrado para comunicarse en las redes sociales, ya que se dará prioridad al aporte de los contenidos en el mismo, así como las actitudes y los valores mostrados en la actividad.

\section{Actividad del alumno:}

- Elabora el mapa conceptual en Powerpoint, en equipo y lo presentan a sus compañeros.

- Participa en el Foro a través de la red social Facebook en un debate en el cual opinan sobre el tema, lo defienden y argumentan su postura, enriqueciendo las opiniones de los compañeros.

Producto: Mapa conceptual del tema Hardware. Deberá incluir los componentes y ejemplos. Dispositivos de Entrada (teclado, mouse, micrófono, scanner, webcam). Dispositivos de Salida 
(impresora, monitor y bocinas). Dispositivos de almacenamiento (memoria principal y memoria secundaria). Dispositivos de Procesamiento ( CPU)

EVALUACIÓN: Rúbrica para evaluar el mapa conceptual. Anexo 3

Rúbrica para evaluar participación en el Foro. Anexo 4

RECURSOS: Libro de texto, Guía de aprendizaje de TIC 1, Computadora, Internet, Libreta, Examen

\section{- CONTEXTO DE APLICACIÓN DE LA ESTRATEGIA DIDÁCTICA}

La estrategia didáctica: "El uso de la red social Facebook para fortalecer en los alumnos el manejo de las TIC'S en la obtención de información y expresión de las ideas"se enmarca en la unidad de aprendizaje: Tecnologías de la Información y la Comunicación I, perteneciente al campo disciplinar de Comunicación, Área Curricular Básica, modalidad presencial, primer semestre.

La propuesta está enmarcada en el campo disciplinar de Comunicación. "Las competencias disciplinares básicas de comunicación están referidas a la capacidad de los estudiantes de comunicarse efectivamente en español...mediante el uso de distintos medios e instrumentos" (Acuerdo $444,2008,7)$. Además, "usarán las tecnologías de la información y la comunicación de manera crítica para diversos propósitos comunicativos" (p. 7)

El alumno adquiere los insumos necesarios sobre el hardware (conceptos, premisas, procedimientos de uso, etc.) a través de una investigación en internet en la web que está disponible en las computadoras del Laboratorio de Cómputo de nuestra preparatoria. Elaboran una lista en la que incluye todos los elementos, características, su descripción y su imagen. Realiza en Powerpoint un mapa conceptual en equipo considerando todos los elementos del hardware de un sistema computacional y lo presentan a sus compañeros de grupo. Además, interactúan con la computadora en internet y en el Foro de discusión de la red social Facebook. El docente presenta las preguntas detonantes en la red social Facebook, las que inician un debate entre los alumnos, los cuáles expresan sus opiniones sobre el tema, lo defienden, argumentan su postura y realizan aportaciones para enriquecer las opiniones de sus compañeros. Los alumnos participan respetando las opiniones de los demás y se les permitirá el uso del código acostumbrado para comunicarse en las redes sociales, ya que se da prioridad al aporte de los contenidos en el mismo, así como las actitudes y los valores mostrados en la actividad. 
En el desarrollo de la secuencia didáctica se pide a los alumnos (productos) que investiguen en internet, realicen una listado con las características de todos los elementos, su descripción e imagen, utilicen esta información en la elaboración de un mapa conceptual; posteriormente participan en un debate en la red social Facebook.

El uso del Facebook en el desarrollo de las actividades fortalece en los alumnos las competencias genéricas, al promover el uso de las TIC'S, ya que a través de ello el estudiante maneja las tecnologías de la información y la comunicación para obtener, procesar, interpretar y expresar sus ideas. Permitiendo el desarrollo de la competencia disciplinar: Utiliza las tecnologías de la información y la comunicación para transmitir información.

- VALORACIÓN DE LAS IMPLICACIONES, POSIBILIDADES Y RETOS QUE PARA LA ENSEÑANZA Y EL APRENDIZAJE CONLLEVA EL DESPLIEGUE DE LA ESTRATEGIA DIDÁCTICA PROPUESTA

Actualmente pensamos que obtener información rápidamente, comunicarnos con miles de personas a la vez a través de Facebook o practicar destrezas de forma automática es algo natural y necesario, pero todos estos hechos eran impensables hace tres décadas.

Las tecnologías basadas en la transmisión de la información a escala mundial, el concepto de desear algo y obtenerlo de inmediato, son cambios en el pensamiento humano que hemos asimilado con la misma celeridad con que han aparecido. Pensar en su significado y en el antes y después nos podría llevar a un conflicto entre la cantidad de información que hemos recibido en algunos años y lo rápido que la hemos asimilado, comparando este hecho con la asimilación de otros conceptos que, a pesar de repetirlos varias veces, aún no podemos memorizar ni recordar. Este proceso ha sido una prueba más de que la necesidad nos hace asimilar hechos que antes habíamos concebido como improbables. La evolución en las tecnologías de la información y la comunicación llevan un ritmo vertiginoso de tendencias y nos conducen a pensar que lo que diseñamos ayer ya no nos sirve para mañana. Estos cambios han provocado modificaciones en las formas de enseñar, puesto que el cambio constante, la rapidez que deseamos para obtener resultados y la gestión de la información, son conceptos que para obtener un resultado satisfactorio han de potenciarse en todos los medios del alumno.

En el México de hoy ya no es suficiente que los maestros de Educación Media Superior (EMS) centremos la acción pedagógica en facilitar la adquisición de conocimientos de la asignatura que impartimos, es indispensable que apoyemos de manera integral la formación de los jóvenes. Nuestra función, va más allá de las prácticas tradicionales de enseñanza en el salón de clases, para adoptar un 
enfoque centrado en el aprendizaje en diversos ambientes, sobre todo ante la Reforma Integral de la Educación Media Superior emprendida para el establecimiento del Sistema Nacional de Bachillerato (SNB) en un marco de diversidad. Acuerdo 447 (2008: 1)

Como aclara Frida Díaz Barriga (1998), (...) el docente se constituye en un mediador en el encuentro del alumno con el conocimiento. En esta mediación el profesor orienta y guía la actividad mental constructiva de sus alumnos, a quienes proporciona ayuda pedagógica ajustada a su competencia. En este sentido el docente realiza diversas acciones en el proceso de enseñar y en el proceso de motivar a los estudiantes para que aprendan. (p.19)

A través del diseño y la implementación de la estrategia: "El uso de la red social Facebook para fortalecer en los alumnos el manejo de las TIC's en la obtención de información y expresión de las ideas", me permitió desarrollar mis competencias docentes, al planificar los procesos de enseñanza y de aprendizaje atendiendo al enfoque por competencias, y los ubiqué en contextos disciplinares, curriculares y sociales amplios. Identifiqué los conocimientos previos y necesidades de formación de los estudiantes y desarrollé estrategias para avanzar a partir de ellas.

Fue necesario contextualizar los contenidos con la vida cotidiana de los estudiantes y la realidad social de la comunidad a la que pertenecen, utilizando la tecnología de la información y la comunicación como una aplicación didáctica y estratégica en la participación de los alumnos en la red social Facebook.

Establecí criterios de evaluación del aprendizaje de los alumnos con base en el enfoque de competencias y los comuniqué de manera clara a los estudiantes a través de la utilización de rúbricas. Comuniqué las observaciones del trabajo realizado a cada uno de los equipos y en particular a los estudiantes de manera constructiva y consistente, y sugiriendo alternativas para su superación. La utilización de Facebook es una de las tantas posibilidades de las nuevas tecnologías que la escuela debe potenciar de forma común, sin valorar el individualismo, que es uno de los peligros potenciales de las nuevas tecnologías. Como maestro de TIC'S interesado por adecuar mi labor docente al contexto de los alumnos, considerando el programa y con la intención de realizar innovaciones en mi planeación, de manera gradual inicié a través de una lluvia de ideas, posteriormente el debate de las mismas de manera grupal, para concluir en lo que hoy realizamos: El debate a través del Facebook. . Anteriormente se realizaba el debate en el salón de clases y de manera verbal. La participación de los estudiantes era muy limitada y no espontánea, además las aportaciones eran superficiales y muy 
poco descriptivas. Al implementar la estrategia de la Red Social Facebook, se presentaron los siguientes cambios en mis alumnos:

a) El $100 \%$ de los alumnos del grupo al menos participaron 2 veces.

b) El $90 \%$ lo hizo en al menos tres ocasiones.

c) Algunas aportaciones (20\%) compartieron creativamente videos, imágenes, presentaciones diseñadas por ellos mismos.

d) Una alumna por iniciativa propia y de manera espontánea, me comentó personalmente: "Hasta que los maestros voltearon a ver lo que verdaderamente nos gusta, platicar a través del Face".

e) En las participaciones de los estudiantes se mostró respeto, un lenguaje claro y coherente aunque usaran un código propio de las charlas de este tipo.

La implementación de la estrategia didáctica: "El uso de la red social Facebook para fortalecer en los alumnos el manejo de las TIC's en la obtención de información y expresión de las ideas" "(...) conllevan otro concepto, el aprendizaje continuado a lo largo de la vida". (Lavid, 2005, p. 35).

Al respecto, menciona María Luisa Carrió Pastor (2007):

La sociedad de la información, es la sociedad del aprendizaje y del conocimiento continuo, ya que los valores se renuevan y cambian constantemente, induciéndonos a innovar en aspectos tradicionales y a adaptarnos a una sociedad cambiante. El mundo laboral exige que nunca dejemos de formarnos, ya que se introducen conceptos y formas de trabajo distintas que hacen que nuestra mentalidad cambie. (p.8)

"Es un reto para los maestros conocer, utilizar y potenciar el uso de las redes sociales y todas sus posibilidades tecnológicas y educativas". (Lavid, 2005, 36).

\section{Conclusiones}

Es nuestra responsabilidad formular una planeación didáctica que propicie el desarrollo de las competencias que se pretenden desarrollar, gestionar los espacios idóneos para el aprendizaje de nuestros alumnos, contemplando los espacios los recursos, interacciones, las actividades, exposiciones e información que se necesitarán para tener un lugar o lugares placenteros que reúnan los requisitos indispensables para la planeación adecuada que permita este desarrollo. 
Para esta planeación se deben tomar en cuenta no solo los contenidos de aprendizaje sino también identificar y diferenciar entre los espacios de un ambiente de aprendizaje adecuados.

El desarrollo de la planeación sirvió para diseñar la trayectoria de aprendizaje de uno de los temas de la unidad de aprendizaje de TIC 1. Para ello es importante que se consideraran los procesos cognitivos de acuerdo a la taxonomía de Marzano. El diseño propiciará el desarrollo de la competencia disciplinar que en este caso es la de comunicación: "Utiliza las tecnologías de la información y la comunicación para investigar, resolver problemas, producir materiales y transmitir información".

La trayectoria de aprendizaje, se define como una secuencia completa de aprendizaje, ya que se puede observar los cuatro momentos que refería Ronal Feo: un inicio, un fin, un desarrollo o proceso, un momento de evaluación, el cual es transversal a toda la trayectoria.

Las actividades de este módulo sirvieron para alinear la estrategia de aprendizaje con la competencia disciplinar que propongo desarrollar en mis estudiantes a través de mi propuesta didáctica.

La estrategia que apoya la trayectoria de aprendizaje del tema "Elementos fundamentales de un sistema computacional" es la participación del grupo en un "debate grupal" en la red social "Facebook". Dicha estrategia busca que "el alumno utilice la tecnologías de la información y la comunicación para investigar, resolver problemas, producir materiales y transmitir información".

El logro de los objetivos la propuesta, el entorno, el clima, y el ambiente de aprendizaje se puede modificar y adecuar a conveniencia. La tarea del docente no se limita solamente al encuentro personal con los estudiantes comprende además otras tareas como: planificación, diagnóstico de alumnos, proyectos para mejoras en el grupo. Asimismo el desarrollo de competencias docentes idóneas para el modelo educativo.

No basta la buena intención como docentes ni la formación previa, en el nuevo modelo educativo mexicano, centrado en el aprendizaje basado en competencias, es indispensable que el docente se preocupe por la planeación didáctica, el ambiente de aprendizaje, particularizando en la organización del espacio educativo y en el tiempo disponible para que, en un curso dado, se logre un nivel de desempeño adecuado en las competencias que se pretende promover

Como docentes requerimos saber planificar los procesos de enseñanza aprendizaje, buscando siempre ubicarlos en contextos sociales amplios y vistos de manera holística, y procurar que el clima 
en el que se realicen coadyuve a la formación integral de los alumnos, todo ello desde la base de sus aspiraciones, motivaciones y necesidades.

El ambiente de aprendizaje y las trayectorias deben ser vistos durante la planeación de una manera horizontal entre sí, pero que al momento de darse las interacciones generen los cruces o transversalidades necesarias para que el suceso educativo o ámbito de aplicación se den de una manera contextualizada.

En nuestra experiencia como docente los métodos que hemos utilizado han funcionado en este último año y medio que hemos trabajado en nuestro plantel bajo el enfoque por competencias. El debate como producto global evidencia que los alumnos dominan el uso de una computadora y el internet, además evidencian que a través de estos recursos se comunican, expresan ideas, investigan, defienden una postura, critican constructivamente a sus compañeros, aceptan sus errores como áreas para mejorar en su aprendizaje.

Para el diseño de la planeación también se tomó en cuenta la evaluación, al diseñar un procedimiento para la evaluación de las competencias se debe tener especial cuidado en no perder de vista la alineación entre productos y metas de aprendizaje (Expresado como competencias u objetivo). EI Concepto de alineación Constructivista de J. Biggs, nos recomienda que para un buen diseño de estrategias de aprendizaje debamos alinear perfectamente lo que se pretende evaluar con los objetivos o metas de aprendizaje y enfocar las actividades o productos de los estudiantes hacia el dominio de las competencias expresadas en los atributos.

Para esta planeación se tuvieron que analizar todos los aspectos tanto de la materia como la competencia que se busca y lo que se pretende evaluar. Para poder hacer una buena planeación didáctica, es importante tomar en cuenta todo lo que se ha desarrollado en el transcurso del diplomado en competencias.

\section{Bibliografía}

Avolio de Cols, S. \&lacolutti, D. (2006). Propuestas para la Enseñanza en la Formación Profesional. En Enseñar y Evaluar en formación por Competencias Laborales. Conceptos y Orientaciones Metodológica. Argentina: Cinterfor.

Biggs, J. (1996). Mejoramiento de la enseñanza mediante la alineación constructiva. De León, D. 
(2010) Evaluación integral de competencias en ambientes virtuales. Universidad de Guadalajara.

Biggs, J. (2010). Calidad del Aprendizaje Universitario. DF, México: SEP-ANUIES.

Carrió Pastor, Ma. Luisa (2007). Ventajas del uso de la tecnología en el aprendizaje colaborativo. Universidad Politécnica de Valencia España.

Chan, M. \& Tiburcio, A. (2000), Guía para la elaboración de materiales orientados al aprendizaje autogestivo. México: Universidad de Guadalajara.

Feo, R. (2009). Estrategias Instruccionales para Promover el Aprendizaje Estratégico en Estudiantes del Instituto Pedagógico de Miranda José Manuel Siso Martínez. Trabajo de grado de Maestría no publicada, Instituto Pedagógico de Miranda José Manuel Siso Martínez, Miranda.Lavid, J. (2005). Lenguaje y nuevas tecnologías. Madrid: Cátedra.

Lavid, Julia (2005). Lenguaje y nuevas tecnologías: Nuevas perspectivas, métodos y herramientas para el Lingüista del siglo XXI. Madrid: Cátedra.

Marzano, R. J. \& Pickering, D. J. (2005). Dimensiones del aprendizaje. Manual para el maestro. México: Ed. ITESO.

SEMS (2008). La reforma integral de la educación media superior. México: SEP.

SEMS. (s.f.). Competencias que expresan el perfil del Docente de la Educación Media Superior. [Documento en PDF]. Recuperado de http://www.sems.gob.mx/aspnv/video/Competencias_que_expresan_el_Perfil_Docente.pdf 